\title{
Cloud in den Wolken
}

Liebe Leserinnen, liebe Leser,

in Diagrammen wird das Internet gerne als eine Kumuluswolke skizziert. Das soll wohl bedeuten, dass es wie eine Wolke beliebig ausgedehnt, veränderlich und intransparent ist, und - vielleicht auch - dass aus der Wolke, analog zu Blitz und Donner, auch Unheil kommen kann. Diese Wolke umschließt demnach eine Maschine globaler Ausdehnung, bestehend aus weltweit verteilten Computern und Übertragungseinrichtungen. Dazwischen fluktuieren elektromagnetische Wellen in Glasfasern, Luft oder leerem Raum. Obwohl ein menschliches Produkt, kann diese Maschine dennoch nicht vom Menschen beherrscht werden. Nicht nur ist sie überaus komplex geraten; sie ist eine sehr ungewöhnliche Maschine. Sie funktioniert nicht ausschließlich nach dem Plan ihres Erfinders, sondern sie wird unablässig von der über die Wolke verteilten menschlichen Intelligenz gelenkt und betrieben. Ähnlich wie das Wetter, erscheint sie von einer Art Laplace'schen Dämons ausgedacht und in Gang gesetzt. Der Dämon betrachtet sie als seine abgeschlossene Schöpfung, ein Kommunikationsnetz und Dienstleistungsmarkt, mit ungezählten menschlichen Akteuren, Teilnehmern, von denen jeder seine eigenen Pläne verfolgt, was sich zu einem Rauschen in den Ohren des Dämons addiert. Jedoch den Teilnehmern gegenüber stellt sich die Wolke anders dar. Jeder sieht sich selbst außerhalb der Wolke. Er tauscht mit ihr sinnvolle Information aus. Sie ist ihm ein Partner auf gleicher Augenhöhe.

Jeder Teilnehmer kann grundsätzlich Dienste empfangen und/oder leisten. Das erfolgt ausschließlich im Medium Information. Die Daten werden von der Wolke entgegengenommen, bearbeitet, gespeichert, verwaltet, übertragen und zugestellt. Das bezeichnet man als Cloud Computing. Die Cloud/Wolke bietet ihren Partner/Teilnehmern wirtschaftliche Vorteile: Ihre gesamten Kapazitäten und Fähigkeiten stehen (im Idealfalle) allen zur Verfügung und sind praktisch unbegrenzt. Ein virtuelles Angebot. Es ist hochgradig modular, flexibel und ökonomisch optimierbar. Die Leistung ist skalierbar; sie kann den Anforderungen und dem Bedarf angepasst werden, ohne dabei empfindlich an Grenzen zu stoßen. Das kann den Bedarf des Teilnehmers gut abdecken. Die Cloud bietet sich inm also als bezahlbares Schlaraffenland an. Jedoch muss er auch damit bezahlen, dass er seine Daten aus der Hand gibt und sie der Cloud überantwortet. Er muss um die Daten bangen, denn er muss mit bösem Willen und Nachlässigkeiten seitens anderer Partner-Teilnehmer und er muss mit Fehlleistungen der Maschine rechnen. Deshalb erwartet er von der Cloud, dass sie inn gegen solche Bedrohungen sichert. Die dazu erforderlichen technischen Maßnahmen lassen sich aus dem Konzept der Cloud ableiten. Eine geschlossene und vollständige Kette von Sicherungen ist das $\mathrm{A}$ und $\mathrm{O}$ des Cloud Computing. Ohne sie kann es keine ordentliche Cloud geben.

Das Handelsgut im Verkehr mit der Cloud sind Informationsdienstleistungen. Die dem Idealbild der Cloud zugeschriebene Flexibilität soll es einem Dienstleistungserbringer möglich machen, Unteraufträge an die Cloud auszugeben. Damit kann die komplettierte Dienstleistung in ihrer Substanz eine Verschachtelung von spezialisierten, willkürlich angeforderten, eventuell flüchtigen Beiträgen sein; in der Regel von Beiträgen unterschiedlicher Rechtspersonen. Daraus ergeben sich nicht nur logistische Probleme. Es ist auch weiterhin für Datenschutz und Rechtssicherheit zu sorgen. Ohne angepasste rechtliche Strukturen bleibt die Cloud definitiv in den Wolken.

Das ist es, liebe Leserinnen und Leser: Meiner Meinung nach muss Cloud Computing so lange in den Wolken bleiben, bis die Herstellerfirmen und Normenausschüsse ihre Hausaufgaben bezüglich der Cloud-Sicherheit gemacht haben und bis angepasste rechtliche Strukturen gefunden sind. Das könnte noch eine Zeit lang auf sich warten lassen.

Mit freundlichen Grüßen, Ihr

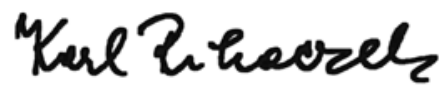

